\section{Acidentes com corpo estranho em menores de 15 anos: análise epidemiológica dos atendimentos em pronto-socorro, internações e óbitos}

\author{
Accidents with foreign bodies in children under \\ 15 years of age: epidemiological analysis \\ of first aid services, hospitalizations, \\ and deaths
}

\footnotetext{
${ }^{1}$ Hospital Universitário, Universidade Estadual de Londrina, Londrina, Brasil. 2 Faculdade de Saúde Pública, Universidade de São Paulo, São Paulo, Brasil. ${ }^{3}$ Departamento de Saúde Coletiva, Universidade Estadual de Londrina, Londrina, Brasil.

Correspondência C. B. G. Martins Hospital Universitário, Universidade Estadual de Londrina.

Rua Chile 185, apto. 103-A, Londrina, $P R$ 86010-220, Brasil. leocris2001@terra.com.br
}

\begin{abstract}
This study aims to analyze accidents involving foreign bodies among children less than 15 years of age residing in Londrina, Paraná State, Brazil, in terms of first aid, hospitalization, and death (2001). Data were obtained from general hospital records and the Municipal Mortality Database. A total of 434 accidents were analyzed, with a $3.7 \%$ hospitalization rate and $0.7 \%$ mortality. Boys predominated (53.7\%), and the incidence rate was highest among children one to three years of age (7.2 per 1,000 children). Foreign body penetration in natural orifices (eyes, nostrils, and ears) accounted for 94\%, inhalation/ingestion of food 2.8\%, inhalation/ingestion of objects $2.5 \%$, and aspiration of gastric contents $0.7 \%$, and these causes accounted for all the deaths. The results contribute to epidemiological knowledge on such accidents and indicate the need to restructure health services in order to decentralize care for less complex injuries, besides emphasizing the need for preventive measures.
\end{abstract}

Foreign Bodies; Child; Domestic Accidents
Christine Baccarat de Godoy Martins 1,2 Selma Maffei de Andrade 3

\section{Introdução}

As causas violentas na infância e a sua prevenção vêm sendo objeto de inúmeros trabalhos nos últimos anos por representarem, atualmente, uma importante causa de morbi-mortalidade infantil $1,2,3,4,5,6,7,8$.

Além das repercussões sociais, econômicas e emocionais na família e na sociedade, os acidentes e violências na infância penalizam nossas crianças e adolescentes em plena fase de crescimento e desenvolvimento 2,9,10,11.

Na medida em que os índices de morbi-mortalidade por causas externas vieram se destacando ao longo das décadas, a necessidade de políticas e estratégias para minimizar este problema de saúde pública mobilizou sociedades e organizações.

Nesse sentido, a Sociedade Brasileira de Pediatria criou, em 1966, o Comitê de Prevenção de Acidentes na Infância 12 e, em 1990, a Convenção sobre os Direitos da Criança e do Adolescente, publicada no Diário Oficial da União em 22 de novembro de 1990, normatizou que "deve-se assegurar a todos os setores da sociedade, em especial aos pais e às crianças, o conhecimento dos princípios básicos de saúde e, entre outros, o de ações de prevenção de acidentes, recebendo apoio para a aplicação destes conhecimentos" 13.

Em outubro de 1998, a Sociedade Brasileira de Pediatria promoveu a Campanha Nacional de Prevenção de Acidentes 5. Mais recentemente, o 
Ministério da Saúde lançou a Política Nacional para Redução da Morbi-Mortalidade por Acidentes e Violências, publicada no Diário Oficial da União em 16 de maio de 2001, com propostas de ações específicas para os gestores federal, estaduais e municipais 14 , cujas diretrizes são: (i) promoção da adoção de comportamentos e de ambientes seguros e saudáveis; (ii) monitoração da ocorrência de acidentes e de violências; (iii) sistematização, ampliação e consolidação do atendimento pré-hospitalar; (iv) assistência interdisciplinar e intersetorial às vítimas de acidentes e de violências; (v) estruturação e consolidação do atendimento voltado à recuperação e à reabilitação; (vi) capacitação de recursos humanos; e (vii) apoio ao desenvolvimento de estudos e pesquisas.

Entre os acidentes infantis mais freqüentes, a penetração de corpo estranho em orifício natural e sua ingestão ou inalação tem sido apontada por vários autores como sendo os cinco principais acidentes que ocorrem na população infantil 3,5,15, atingindo principalmente os lactentes e menores de um ano, além de se constituir um acidente potencialmente fatal por causar, muitas vezes, obstrução das vias respiratórias 16,17.

Vários trabalhos nacionais $17,18,19,20$ e internacionais 21,22,23 apontam a presença de corpo estranho como importante causa de morbidade e mortalidade entre as crianças.

Apesar dos estudos chamarem a atenção para a ocorrência desse tipo de evento na população infantil, pouco conhecimento se tem acerca dos objetos envolvidos nesse acidente e da evolução das vítimas. Nesse sentido, estudar as causas e circunstâncias desse agravo se torna essencial a fim de ampliar o conhecimento epidemiológico acerca desses eventos e formar um diagnóstico que contribua para o desenvolvimento e implementação de programas voltados para a prevenção dessa importante causa de atendimento e óbito infantil.

O presente trabalho teve o objetivo de estudar a ocorrência dos acidentes por penetração em orifício natural, ingestão e inalação de corpo estranho entre menores de 15 anos, residentes em Londrina, Paraná, Brasil, que foram atendidos em serviços de emergência e internação, ou que morreram devido a estas causas em 2001.

\section{Metodologia}

A pesquisa se constituiu em um estudo transversal com dados retrospectivos e a população pesquisada foi composta por menores de 15 anos de idade, residentes no Município de Londrina, vítimas de acidentes por penetração de corpo estranho no/ou através do olho e orifício natural (código W44 da Classificação Internacional de Doenças, 10a revisão - CID-10) 24 , inalação de conteúdo gástrico (código W78), inalação e ingestão de alimentos causando obstrução do trato respiratório (código W79), inalação e ingestão de outros objetos com obstrução do trato respiratório (código W80), ocorridos no período de $1^{\circ}$ de janeiro a 31 de dezembro de 2001, que foram atendidos em serviços de emergência ou internação, ou que morreram devido a estes eventos em um prazo de até um ano após o acidente ou ato violento, conforme preconiza a CID-10.

Os dados de morbidade (atendimento de emergência/urgência e internação) foram obtidos em todos os serviços hospitalares de emergência e internação de Londrina que prestam atendimento a crianças vítimas de acidentes, e os dados de mortalidade foram coletados no Núcleo de Informação em Mortalidade (NIM) da Prefeitura de Londrina, considerando que o óbito poderia ter ocorrido sem que o atendimento houvesse sido prestado por serviços de saúde. Utilizou-se um instrumento previamente testado para transcrição de dados dos prontuários selecionados.

Os dados de morbidade foram processados eletronicamente usando-se o programa computacional Epi Info, versão 6.0.d (Centers for Disease Control and Prevention, Atlanta, Estados Unidos), excluindo-se os atendimentos duplicados referentes a um mesmo acidente. Os óbitos que ocorreram sem assistência foram incluídos com vistas a formar um único banco de dados de morbi-mortalidade. Foi realizada uma criteriosa verificação dos casos, eliminando-se os registros repetidos a fim de evitar a duplicação de dados. Foi realizada exaustiva verificação de inconsistências por meio do cruzamento e verificação de dados. Tendo por base a população estimada na mesma faixa etária e ano, calculou-se o coeficiente de incidência utilizando-se um denominador de mil crianças.

O presente estudo foi aprovado pelo Comitê de Ética em Pesquisa da Universidade Estadual de Londrina (parecer CEP 126/02, de 21 de outubro de 2002).

\section{Resultados}

Foram estudados 434 casos de acidentes com corpo estranho (penetração em orifício natural, inalação e ingestão) em menores de 15 anos no ano de 2001.

A penetração de corpo estranho em orifício natural (olho, fossas nasais e conduto auditivo) representou $94 \%$, seguida pela inalação e inges- 
tão de alimentos $(2,8 \%)$, pela inalação e ingestão de outros objetos $(2,5 \%)$ e pela inalação de conteúdo gástrico $(0,7 \%)$ (Tabela 1$)$.

Quanto ao nível de atendimento e evolução das vítimas, os acidentes por corpo estranho foram responsáveis por 393 atendimentos exclusivamente em pronto-socorro (95,6\%), por 16 internações $(3,7 \%)$ e três óbitos $(0,7 \%)$. As internações ocorreram, predominantemente, devido à penetração de corpo estranho no/ou através do olho e orifício natural (87,5\% das internações) e por inalação/ingestão de objeto (12,5\%). Os três óbitos ocorreram por aspiração de conteúdo gástrico, dos quais dois se deram sem assistência médica. Para cada óbito, ocorreram 131 atendimentos de pronto-socorro e aproximadamente cinco internações.

Houvepredomínio do sexomasculino (53,7\%), numa relação de 1,2 caso masculino para cada caso feminino. A faixa etária com maior número de casos foi a de 1 a 3 anos de idade $(46,3 \%)$

(Tabela 2).
Ao calcular o coeficiente de incidência para os acidentes com corpo estranho, observou-se que a faixa etária com maior risco é a de 1 a 3 anos de idade, com coeficiente total de 7,2 por mil crianças, com incidência de 7,6 para mil meninas e de 6,7 para mil meninos. O coeficiente masculino é superior ao feminino na maioria das faixas etárias, exceto entre os menores de 1 ano e no grupo etário de 1 a 3 anos (Figura 1).

Conhecer os agentes responsáveis pelos acidentes com corpo estranho permite agir diretamente sobre eles, retirando-os do ambiente da criança, evitando ferimentos e lesões desnecessárias.

Nesse sentido, a Tabela 3 distribui os acidentes por corpo estranho segundo os agentes envolvidos. Observa-se que as moedas, as bolinhas/botões/tampinhas, os grãos/sementes e as pecinhas de brinquedo foram os agentes mais freqüentes.

Tabela 1

Distribuição dos menores de 15 anos vítimas de acidentes com corpo estranho, segundo o tipo de acidente. Londrina, Paraná, Brasil, 2001.

\begin{tabular}{lcc}
\hline Tipo de acidente segundo o código da CID-10 & $\mathbf{n}$ & $\%$ \\
\hline W44 - Penetração de corpo estranho no/ou através de olho e orifício natural & 408 & 94,0 \\
W78 - Inalação de conteúdo gástrico & 3 & 0,7 \\
W79 - Inalação e ingestão de alimentos causando obstrução do trato respiratório & 12 & 2,8 \\
W80 - Inalação e ingestão de outros objetos com obstrução do trato respiratório & 11 & 2,5 \\
Total & 434 & 100,0 \\
\hline
\end{tabular}

CID-10: Classificação Internacional de Doenças, 10ạ revisão 24.

Tabela 2

Distribuição dos menores de 15 anos vítimas de acidentes por corpo estranho, segundo o sexo e a faixa etária. Londrina, Paraná, Brasil, 2001

\begin{tabular}{|c|c|c|c|c|c|c|}
\hline \multirow{2}{*}{$\begin{array}{l}\text { Faixa etária } \\
\text { (anos) }\end{array}$} & \multicolumn{2}{|c|}{ Masculino } & \multicolumn{2}{|c|}{ Feminino } & \multicolumn{2}{|c|}{ Total } \\
\hline & $\mathbf{n}$ & $\%$ & $\mathrm{n}$ & $\%$ & $\mathrm{n}$ & $\%$ \\
\hline$<1$ & 7 & 3,0 & 8 & 3,9 & 15 & 3,5 \\
\hline $1-3$ & 97 & 41,6 & 104 & 51,7 & 201 & 46,3 \\
\hline $4-6$ & 64 & 27,5 & 55 & 37,4 & 119 & 27,4 \\
\hline $7-9$ & 34 & 14,6 & 22 & 10,9 & 56 & 12,9 \\
\hline $10-14$ & 31 & 13,3 & 12 & 6,0 & 43 & 9,9 \\
\hline Total & 233 & 53,7 & 201 & 46,3 & 434 & 100,0 \\
\hline
\end{tabular}


Figura 1

Distribuição dos coeficientes de incidência de acidentes por corpo estranho em menores de 15 anos. Londrina, Paraná, Brasil, 2001.

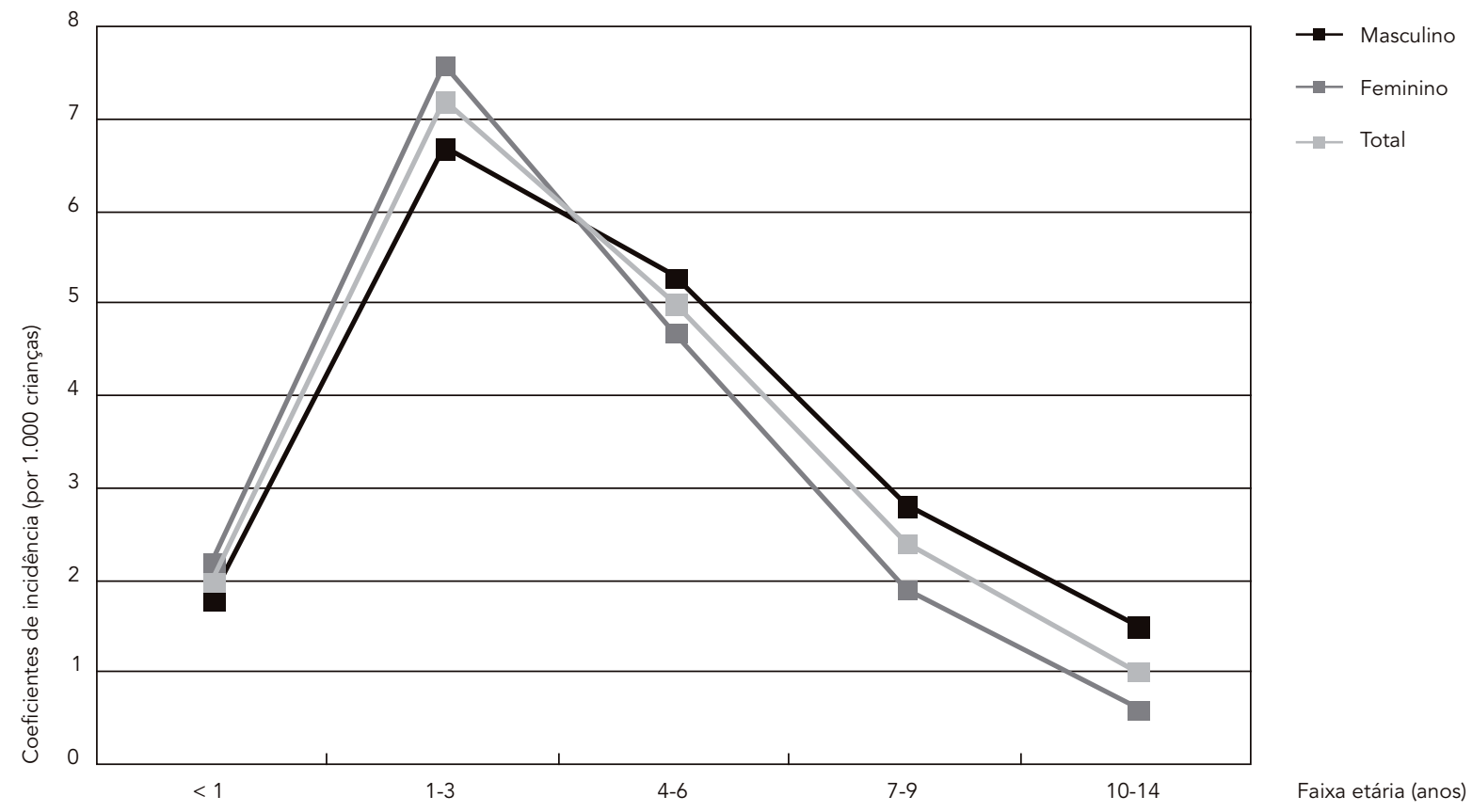

Tabela 3

Distribuição dos menores de 15 anos vítimas de acidentes por corpo estranho, segundo o agente envolvido. Londrina, Paraná, Brasil, 2001.

\begin{tabular}{lcc}
\hline Agente envolvido & $\mathbf{n}$ & $\%$ \\
\hline Alimento & 12 & 2,8 \\
Conteúdo gástrico & 3 & 0,7 \\
Cotonete & 10 & 2,3 \\
Bijuteria & 15 & 3,5 \\
Pedaços de borracha/Esponja/Barbante/Linha/Bexiga/Plástico & 11 & 2,5 \\
Bolinha/Botão/Tampinha de medicamento e produto de limpeza & 32 & 7,4 \\
Cola e giz & 11 & 2,5 \\
Caneta e lápis & 7 & 1,6 \\
Grãos e sementes & 32 & 7,4 \\
Inseto & 3 & 0,7 \\
Moeda & 50 & 11,5 \\
Palito (fósforo, pirulito) & 8 & 1,8 \\
Parafuso/Prego/Pedra & 11 & 2,5 \\
Pecinha de brinquedo & 30 & 6,9 \\
Prendedor de chupeta & 4 & 0,9 \\
Agente não especificado & 195 & 44,9 \\
Total & 434 & 100,0 \\
\hline
\end{tabular}




\section{Discussão}

Faz-se necessário destacar que o presente estudo abrange apenas as crianças atendidas em pronto-socorro ou que foram internadas, ou que morreram, tratando-se, portanto, de uma incidência mínima, referente apenas àquela parcela que chega aos serviços de atendimento de emergência ou que morreram sem assistência.

O número de casos de acidentes com corpo estranho encontrados na presente investigação correspondeu a $4,9 \%$ de todos os atendimentos hospitalares, internações e óbitos infantis por causas externas (acidentes e violências), no mesmo período e faixa etária 25. Estudos nacionais 3,5,15 também identificaram os acidentes por corpos estranhos entre as cinco primeiras causas de acidentes entre crianças, com percentuais que variaram de $2,8 \%$ a 9,3\% em relação ao total de acidentes infantis ocorridos na população estudada.

Quanto ao nível de atendimento e evolução das vítimas, não foi possível comparar a presente casuística com outros estudos em virtude da escassez de trabalhos com esta análise. Vários autores trazem a proporção de atendimentos de pronto-socorro, internação e óbito em relação ao total de acidentes em serviços específicos, mas não exclusivamente quanto aos acidentes com corpo estranho 3,5. Taxas semelhantes de internação e óbito foram encontradas em investigações como a de Filócomo et al. ${ }^{5}$, que verificaram 95,7\% de atendimentos de pronto-socorro em crianças vítimas de acidentes e uma taxa de internação igual a $4 \%$. Baracat et al. 3 também encontraram $97,1 \%$ de atendimentos em pronto-socorro e um percentual de $0,1 \%$ para os óbitos por acidentes em crianças de 0 a 14 anos.

Apesar da taxa de internação de apenas 3,7\%, no presente estudo, a característica do acidente estudado permite considerar a gravidade do evento por causar obstrução das vias aéreas, com grande risco de óbito, presente em $0,7 \%$ da população de estudo, tornando-se necessário o desenvolvimento de campanhas de conscientização e outras medidas de prevenção.

Diante da alta proporção de atendimento de pronto-socorro, é importante ressaltar que grande parte dos atendimentos neste nível de atenção poderia ser evitada por meio da adoção de medidas preventivas efetivas 26 , reduzindo os gastos hospitalares com esses eventos e as situações de estresse vividas pela criança e sua família. Acrescenta-se, ainda, a necessidade de ampliar e capacitar o atendimento nos serviços de atenção primária no sentido de desafogar a demanda nos prontos-socorros.

Os óbitos sem assistência médica, por sua vez, ocorridos no local do acidente ou a caminho do serviço de urgência, suscitam a necessidade de serviços pré-hospitalares de rápido acesso e de campanhas de orientação quanto aos primeiros procedimentos a serem adotados na presença de tais acidentes. O uso dos diversos meios de comunicação e a realização de campanhas de alerta quanto ao risco e incidência desses acidentes podem contribuir para reduzir essa causa de morbi-mortalidade infantil.

O predomínio do sexo masculino entre as vítimas de acidentes com corpo estranho pode ser comparado com outros estudos que demonstram maior proporção deste sexo entre as vítimas de acidentes infantis 1,3,4,5,15. A freqüência mais elevada no sexo masculino vem sendo discutida e justificada pela diferença de atividades entre os dois sexos, além da maior vigilância sobre as crianças do sexo feminino.

Em relação à idade, a inexistência de outros trabalhos brasileiros com estimativas da taxa de incidência dificultou a comparação. As investigações existentes não se referem à população total, restringindo-se a instituições específicas e apresentando dados referentes a amostras de menor tamanho. Os estudos de Ballesteros et al. 8, de Filócomo et al. 5 e de Baracat et al. 3 , por exemplo, apesar de tratarem dos acidentes em geral, identificaram a penetração de corpos estranhos em orifício natural como o acidente mais freqüente nas idades de um a seis anos. Bittencourt \& Camargos 16 também apontam que a maioria das vítimas de aspiração de corpo estranho são lactentes e crianças nos primeiros anos de vida. Estudo retrospectivo com 92 pacientes com aspiração de corpo estranho também revela maior freqüência $(46,4 \%)$ entre crianças de um e dois anos 27 . Os menores de três anos também são apontados por Oliveira et al. 20 como as vítimas mais freqüentes de corpo estranho em vias aéreas.

A incidência maior de acidentes com corpo estranho na faixa etária de um a três anos, possivelmente, está associada às características do seu desenvolvimento neuro-psicomotor (imaturidade física e mental, inexperiência, incapacidade para prever e evitar situações de perigo, curiosidade, tendência a imitar comportamentos adultos, falta de noção corporal e de espaço, pouca coordenação motora, fase oral que faz com que as crianças menores levem os objetos à boca) e características da personalidade de algumas crianças (hiperatividade, agressividade, impulsividade e distração), além de particularidades orgânicas ou anatômicas, tais como deficiência física e/ou mental 6,12,28. A criança, nesse contexto, encontra-se muitas vezes indefesa e vulnerável.

Além dessas características, alguns autores apontam que os acidentes guardam relação com uma ampla rede de fatores, tais como as condi- 
ções ambientais, físicas, culturais e sociais da família: o estilo de vida dos pais, as condições de vida e de trabalho, a taxa de urbanização, a marginalidade, o desemprego, a desigualdade social, a miséria, o nível de educação, as condições impróprias de moradia, a vigilância insuficiente, entre outros 6,12,29,30,31.

Estudos demonstram que a maioria dos acidentes ocorre em casa 5,32 pelo fato de a residência apresentar grande número de objetos e situações de riscos para a ocorrência destes eventos. Além disso, em muitos casos, pode haver uma supervisão inadequada da criança, pelo simples fato de o adulto responsável por seu cuidado desenvolver outras atividades ao mesmo tempo. É importante ressaltar, todavia, que apenas a presença do adulto não impede que os acidentes ocorram, como mostrou o estudo de Filócomo et al. 5 , no qual apenas $4,3 \%$ das crianças estavam sozinhas, $43,4 \%$ estavam na presença dos pais, $22,2 \%$ na de amigos e 8,3\% na presença da professora.

Considerando que os acidentes com corpo estranho ocorrem, em grande parte, com objetos presentes no lar, pode-se compreender que a modificação do ambiente doméstico constitui uma forma importante de prevenção, bem como a adoção de comportamentos seguros e vigilância adequada por parte dos pais e responsáveis. Medidas de orientação quanto às etapas do desenvolvimento da criança e seus respectivos riscos de acidentes são essenciais diante desse contexto.

Os diferentes corpos estranhos identificados neste estudo nos levam a perceber que são objetos de nosso cotidiano, estando facilmente ao alcance da criança. Boletim da Sociedade Brasileira de Pediatria, divulgado em 1998 33, relata que balas, uvas, nozes, chupetas pequenas, bolas pequenas, pecinhas de brinquedo e balões foram os maiores responsáveis pela asfixia e sufocamento em crianças. A moeda também foi identificada em outros levantamentos como o corpo estranho mais comum em esôfago de crianças 18,34.

As moedas, botões, grãos, sementes, pecinhas de brinquedo, prendedores de chupeta, bijuterias, tampinhas e inúmeros outros objetos passam despercebidos pelo adulto, mas são verdadeiros atrativos para as crianças. Na sua fase de exploração e descoberta do corpo, a criança introduz esses objetos em fossas nasais e condutos auditivos, chegando a degluti-los numa verdadeira experiência corporal. Para evitar que tais episódios ocorram, torna-se importante os cuidados com o ambiente, livrando-o dos agentes acima listados e adequando-o às necessidades da criança.

A grande freqüência de agente não especificado desperta para a necessidade de melhoras no registro e na qualidade das informações 35 . A criação e implantação de um protocolo de atendimento às crianças vítimas de acidentes e o treinamento dos profissionais na determinação de suas circunstâncias podem contribuir para o conhecimento epidemiológico dessa importante causa de morbi-mortalidade, direcionando melhor o planejamento das ações de controle e prevenção. A abordagem do tema nos cursos de graduação também torna-se fundamental, buscando sensibilizar os alunos quanto à importância do registro correto e completo da informação.

A presente investigação, ao delinear o perfil municipal de morbi-mortalidade por acidentes com corpo estranho entre os menores de 15 anos, com análise detalhada segundo o nível de atendimento, agentes envolvidos, com coeficientes de incidência nas diferentes faixas etárias e sexos, permite aos planejadores analisar tendências, priorizar ações que contemplem a prevenção e atenção às vítimas desses eventos e desenvolver estratégias para a reestruturação dos serviços, a fim de tornar mais eficiente o gerenciamento dos recursos, pois para Mello-Jorge et al. 36 (p. 520), "os dados de saúde permitem formar uma idéia aproximada da magnitude e complexidade do problema".

\section{Conclusão}

Os resultados encontrados neste estudo alertam para o perigo que constituem os objetos pequenos que a criança possa introduzir nas fossas nasais, no conduto auditivo e até mesmo ingerir, ocasionando risco de vida e trauma para retirada do corpo estranho. Os botões, grãos, sementes, moedas, tampinhas, entre inúmeros outros objetos, precisam ser mantidos longe do alcance da criança. Os brinquedos devem ser examinados cuidadosamente, pois pecinhas que se soltam com facilidade também são responsáveis por esse tipo de acidente. Muitas vezes, a mãe ou responsável só percebe o acidente mais tarde, quando a criança apresenta dor e edema devido à inflamação do conduto auditivo ou fossas nasais, ou quando a criança apresenta dificuldade respiratória por obstrução das vias aéreas.

O fato dos acidentes por corpo estranho acontecerem, em grande parte, no ambiente doméstico sugere a modificação do mesmo como forma efetiva de prevenção, além da supervisão direta sobre a criança.

Os resultados nos levam a acreditar que por meio de ações e orientações específicas aos pais e cuidadores, é possível reduzir em grande parte os acidentes por corpo estranho. Para Oliveira \& Ribeiro 37 (p. 249), a educação em saúde cons- 
titui-se "uma importante estratégia para instrumentalizar os indivíduos para o autocuidado e a obtenção do melhor potencial de saúde e qualidade de vida".

Os resultados encontrados contribuem para ampliar o conhecimento epidemiológico acerca de tais eventos e apontam para a necessidade de medidas preventivas.

Sugerimos, ainda, novos estudos que venham complementar lacunas do conhecimento e contribuir para a implementação de ações preventivas e de controle.

\section{Resumo}

Analisou-se os acidentes com corpo estranho entre menores de 15 anos, residentes em Londrina, Paraná, Brasil, atendidos em serviços de emergência/internação ou que morreram por estas causas, em 2001. Os dados foram obtidos nos hospitais gerais e no Núcleo de Informação em Mortalidade do município. Foram estudadas 434 crianças vítimas de acidentes com corpo estranho, revelando uma taxa de internação de 3,7\% e uma taxa de óbito de 0,7\%. Houve predomínio do sexo masculino $(53,7 \%)$ e o maior coeficiente foi na faixa etária de 1 a 3 anos (7,2 por mil crianças). A penetração de corpo estranho em orifício natural (olho, fossas nasais e conduto auditivo) representou $94 \%, a$ inalação/ingestão de alimentos ocorreu em 2,8\%, a inalação/ingestão de objetos em $2,5 \%$ e a inalação de conteúdo gástrico em $0,7 \%$ dos casos, sendo responsável por todos os óbitos. A presente investigação pode contribuir no sentido de permitir aos planejadores analisar tendências, priorizar ações que contemplem a prevenção e atenção às vítimas desses eventos e desenvolver estratégias para a reestruturação dos serviços a fim de tornar mais eficiente o gerenciamento dos recursos.

Corpos Estranhos; Criança; Acidentes Domésticos

\section{Colaboradores}

Todos os autores participaram da concepção do trabalho, da análise e interpretação dos dados, de sua redação, da revisão crítica e da leitura e aprovação da versão final.

\section{Referências}

1. Del Ciampo LA, Ricco RG, Muccillo G. Acidentes: sabemos preveni-los? Pediatria (São Paulo) 1997; 19:263-6.

2. Souza LJEX, Barroso MGT. Revisão bibliográfica sobre acidentes com crianças. Rev Esc Enferm USP 1999; 33:107-12.

3. Baracat ECE, Paraschin K, Nogueira RJN, Reis MC, Fraga AMA, Sperotto G. Acidentes com crianças e sua evolução na região de Campinas, SP. J Pediatr (Rio J) 2000; 76:368-74.

4. Barros MDA, Ximenes R, Lima MLC. Mortalidade por causas externas em crianças e adolescentes: tendências de 1979 a 1995. Rev Saúde Pública 2001; 35:142-9.

5. Filócomo FRF, Harada MJS, Silva CV, Pedreira MLG. Estudo dos acidentes na infância em um prontosocorro pediátrico. Rev Latinoam Enf 2002; 10:41-7.

6. Fonseca SS, Victora CG, Halpern R, Barros AJD, Lima RC, Barros FC, et al. Fatores de risco para injúrias acidentais em pré-escolares. J Pediatr (Rio J) 2002; 78:97-104.

7. Hippisley-Cox J, Groom L, Kendrick D, Coupland C, Webber E, Savelyich B. Cross sectional survey of socioeconomic variations in severity and mechanism of childhood injuries in Trent, 1992-1997. BMJ 2002; 324:1132.

8. Ballesteros MF, Schieber RA, Gilchrist J, Holmgreen P, Annest JL. Differential ranking of causes of fatal versus non-fatal injuries among US children. Inj Prev 2003; 9:173-6.

9. Blank D. Conceitos básicos e aspectos preventivos gerais. In: Comitê de Acidentes na Infância, organizador. Manual de acidentes na infância e adolescência. São Paulo: Sociedade Brasileira de Pediatria; 1994. p. 2-13. 
10. Tursz A, Crost M. Sequelae after unintentional injuries to children: an exploratory study. Inj Prev 2000; 6:209-13.

11. Mello-Jorge MHP, Gotlieb SLD, Laurenti R. A saúde no Brasil: análise do período 1996 a 1999. Brasília: Organização Pan-Americana da Saúde; 2001.

12. Del Ciampo LA, Ricco RG. Acidentes na infância. Pediatria (São Paulo) 1996; 18:193-7.

13. Brasil. Convenção sobre os Direitos da criança e adolescente de 1990, Decreto no ${ }^{\circ}$ 99.710. Diário Oficial da União 1990; 22 nov.

14. Ministério da Saúde. Política nacional de redução da morbi-mortalidade por acidentes e violências: Portaria MS/GM no. 737 de 16/05/2001. Diário Oficial da União 2001; 18 mai.

15. Harada MJCS, Botta MLG, Kobata CM, Szauter IH, Dutra G, Dias EC. Epidemiologia em crianças hospitalizadas por acidentes. Folha Méd 2000; 119:43-7.

16. Bittencourt PFS, Camargos PAM. Aspiração de corpos estranhos. J Pediatr (Rio J) 2002; 78:9-18.

17. Fraga JC, Alexandra FP, Komlos M, Takamatu EE, Luciano GC, Contelli FHA. Remoção de corpo estranho da via aérea de criança por broncoscopia através de trauqueotomia ou traqueostomia. J Pediatr (Rio J) 2003; 79:369-72.

18. Queiroz, VF, Rocha RCA, Bastos ELS, Soares FV, Salvi JA, Silvado RA. Corpo estranho no esôfago. GED Gastroenterol Endosc Dig 2002; 21:49-54.

19. Lima JAB, Fischer GB, Felicetti JAF, Penna CN, Ludwig E. Aspiração de corpo estranho na árvore traqueobrônquica em crianças: avaliação de seqüelas através de exame cintilográfico. J Pneumol 2000; 26:345-51.

20. Oliveira CF, Almeida JFL, Troster EJ. Complicações de aspiração de corpo estranho traqueobrônquico em crianças: relato de 5 casos e revisão da literatura. Rev Hosp Clin Fac Med Univ São Paulo 2002; 57:108-11.

21. Bekhof J, Norbruis OF, Scheenstra R, Weerd W. Active management of children after ingestion of a button battery. Ned Tijdschr Geneeskd 2005; 149:1429-33.

22. Cauchi JA, Shawis RN. Multiple magnet ingestion and gastrointestinal morbidity. Arch Dis Child 2002; 87:539-40.

23. Tan HK, Brown K, McGill T, Kenna MA, Lund DP, Healy GB. Airway foreign bodies (FB): a 10-year review. Int J Pediatr Otorhinolaryngol 2000; 56:91-9.

24. Organização Mundial da Saúde. Classificação internacional de doenças e de problemas relacionados à saúde - 10a revisão. 8a Ed. São Paulo: Centro Colaborador da OMS para a Classificação de Doenças em Português/Edusp; 2000.
25. Martins CBG, Andrade SM. Causas externas entre menores de 15 anos em cidade do Sul do Brasil: atendimentos em pronto-socorro, internações e óbitos. Rev Bras Epidemiol 2005; 8:194-204.

26. Towner E, Dowswell T, Jarvis S. Updating the evidence. A systematic review of what works in preventing childhood unintentional injuries: part I. Inj Prev 2001; 7:161-4.

27. Marques MPC, Couto FD, Fim LA, Nogueirol RB, Oliveira VS. Broncoscopia rígida: a terapia do corpo estranho de vias aéreas. Rev Bras Otorrinolaringol 1997; 63:551-6.

28. Larsson J, Aurelius G. Accidents in childhood: relation to psychosocial condition and mental development. Acta Paediatr 1996; 85:285-91.

29. Minayo MCS. A violência na adolescência: um problema de saúde pública. Cad Saúde Pública 1990; 6:278-92.

30. Minayo MCS. A violência social sob a perspectiva da saúde pública. Cad Saúde Pública 1994; 10 Suppl 1:7-18.

31. Lima MLC, Ximenes R. Violência e morte: diferenciais da mortalidade por causas externas no espaço urbano do Recife, 1991. Cad Saúde Pública 1998; 14:829-40.

32. Mesquita Filho M. Vítimas de causas externas atendidas em serviço de urgência e emergência - subsídios ao desenvolvimento de sistema de informações [Tese de Doutorado]. São Paulo: Faculdade de Saúde Pública, Universidade de São Paulo; 2003.

33. Maciel W. Campanha nacional de prevenção de acidentes na infância e adolescência. Boletim da Sociedade Brasileira de Pediatria 1998; 73:4-5.

34. T-Ping C, Nunes CA, Guimarães GR, Vieira JP, Weckx LL, Borges TJ. Accidental ingestion of coins by children: management at the ENT Department of the João XXIII Hospital. Rev Bras Otorrinolaringol 2006; 72:470-4.

35. Drumond Jr. M, Lira MMTA, Freitas M, Nitrini TMU, Shibao K. Avaliação da qualidade das informações de mortalidade por acidentes não especificados e eventos com intenção indeterminada. Rev Saúde Pública 1999; 33:273-80.

36. Mello-Jorge MHP, Marques MB, Meller JL, Stein RJ. Mortes violentas em menores de 15 anos no Brasil. Bol Oficina Sanit Panam 1990; 100:590-606.

37. Oliveira AAP, Ribeiro MO. O cuidar da criança de/na rua na perspectiva dos graduandos de enfermagem. Texto \& Contexto Enferm 2006; 15:246-53.

Recebido em 08/Out/2007

Versão final reapresentada em 08/Jan/2008

Aprovado em 12/Fev/2008 